\title{
Capitalismo, neoliberalismo y ambiente. Las inconsistencias teóricas de la ciencia económica
}

\author{
Gesly Aníbal Bonilla Landaverry \\ Ingeniero Agrónomo, Maestro en Ciencias en Gestión Ambiental y \\ Doctor en Ciencias Políticas y Sociología \\ gesly77@hotmail.com \\ Centro Universitario de Sur Oriente \\ Universidad de San Carlos de Guatemala \\ Otto René Solís Méndez \\ Licenciado en Economía \\ Centro Universitario de Sur Oriente \\ Universidad de San Carlos de Guatemala
}

Fecha de recepción: 08/06/2015 Fecha de aceptación: 17/08/2015

\begin{abstract}
Resumen
La Economía se ha convertido en la ciencia que ha llegado a dominar la racionalidad humana. Se han desarrollado modelos para su impulso, predominando en la actualidad el neoliberal, basado en la economía neoclásica liberal, que retoma las bases teóricas del equilibrio general formuladas por los economistas neoclásicos a inicios de 1900 y los principios del libre mercado. Desde el nacimiento de la ciencia económica, Adam Smith trató de explicar con la metáfora de "la mano invisible" en su obra El origen y naturaleza de la riqueza de las Naciones publicada en 1776, que el mercado asigna los recursos eficientemente. Sin embargo, han pasado más de 200 años y no se ha podido demostrar, en la práctica, que este principio se cumpla. Por el contrario, en su aplicación, el sistema económico dominante y el modelo económico neoliberal, están causando efectos negativos sobre las sociedades más pobres de los países latinoamericanos, y es que desde sus fundamentos teóricos existen inconsistencias y contradicciones que no permiten en la práctica, obtener el equilibrio económico y armonía social deseada.
\end{abstract}

\section{Palabras clave}

Teoría del equilibrio general, capitalismo, neoliberalismo, inconsistencias teóricas, economía

\begin{abstract}
Economics has become the science that has come to dominate human rationality. Models have been developed to its momentum, dominating today the neoliberal, based on the liberal neoclassical economics, which incorporates the theoretical foundations of general equilibrium, where neoclassical economists made by the beginning of 1900 based on the principles of free market. Since the birth of economics, Adam Smith tried to explain the metaphor of the "invisible hand" in his work The Origin and Nature of the Wealth of Nations published in 1776, that the market allocates resources efficiently. However, it has been more than 200 years and has not been able to demonstrate in practice that this principle is fulfilled. On the contrary, in its application, the dominant economic system and the neoliberal economic model, are causing negative effects on poorer countries of Latin American societies, it is that since its theoretical inconsistencies and contradictions are not allowed in practice, obtain the desired economic balance and social harmony.
\end{abstract}

\section{Keywords}

General equilibrium theory, capitalism, neoliberalism, theoretical inconsistencies, economy 


\section{Introducción}

El impulso natural hacia el comercio y el intercambio siempre ha existido desde el inicio de la humanidad. En su momento, estas actividades fueron acentuadas y fomentadas por las Cruzadas que se organizaron en Europa occidental desde el siglo XI hasta el siglo XIII. Así surge en Venecia el capitalismo, que luego se trasladó a Florencia en la época medieval (Nadal, 2008), para posteriormente tomar su mayor impulso durante la Revolución Francesa, Industrial y la Independencia de los Estados Unidos (Monterroso, 2007). Estos procesos cambiaron de forma drástica a las sociedades, facilitando la aparición de los Estados Nacionales que proporcionaron las condiciones necesarias para el crecimiento y evolución de la economía, encontrando en el lluminismo y la Reforma protestante, un espíritu capitalista sobre los pueblos europeos entre los siglos XVII y XVIII como su principal motor impulsor para desarrollar sus economías (Weber, 2006).

En la actualidad, el capitalismo usa un modelo de desarrollo económico basado en la apertura de mercados, al cual muchos economistas han denominado Neoliberalismo ${ }^{1}$. Sin embargo, los efectos que ha producido el impulso del mismo sobre las poblaciones más pobres y áreas rurales en los países latinoamericanos son desastrosos (Ramírez, 2008), causando estragos en la desestructuración económica de los campesinos (McAfee, 2003).

Este artículo es un intento de mostrar que la economía dominante y el modelo de mercados abiertos o "Neoliberalismo" presentan una serie de inconsistencias en sus fundamentos teóricos del equilibrio general, a pesar que

1 El término neoliberalismo es una abreviación de neoclassical liberalism (liberalismo neoclásico), y hace referencia a una política económica que considera contraproducente el excesivo intervencionismo estatal en materia social o en la economía y defiende el libre mercado capitalista como mejor garante del equilibrio institucional y el crecimiento económico de un país. desde hace más de 200 años se ha querido demostrar la eficiencia del libre mercado.

Para Screpanti (1997), el trabajo de Leon Walras $^{2}$ sobre la Teoría General del Equilibrio fue muy importante, pero a la vez reconoce que presenta inconsistencias y contradicciones, porque en teoría los consumidores y productores están sujetos a precios sobre los que nadie tiene poder para influir y en la práctica del libre mercado existen los monopolios, que son una verdadera amenaza al sistema económico (Galbraith, 1979:39). Es como este tipo de contradicciones que, en la práctica, el modelo neoliberal está causando efectos negativos sobre las sociedades más desiguales (Ramírez, 2008), repercutiendo además en el ambiente (Martínez, 2008). Por eso, como científicos y productores de conocimiento se debe tener claro que la práctica de un fenómeno natural o social debe fundamentarse con una teoría consistente, la cual no debe presentar contradicción alguna entre el pensamiento y las leyes de la realidad (Hessen, 1990).

Este trabajo se enfoca en hacer una crítica a la ciencia económica que ha evolucionado para dominar la racionalidad humana (Leff, 2004), la cual se fundamenta en la Teoría General del Equilibrio (TGE), usando el Modelo de Mercados Abiertos (MECA) y aplica estrategias como los Tratados de Libre Comercio (TLC) para ser funcional. Todo esto deberá ser motivo de reflexión, en principio, por la comunidad cientí-

2 Léon Walras (Évreux, Francia, 16 de diciembre 1834 - Clarens-Montreux, Suiza, 5 de enero 1910), economista francés de la Escuela de Lausana. Es considerado a menudo el fundador de la economía matemática. Walras fue el primero en analizar y describir el equilibrio general de la competencia perfecta, para explicar cómo los precios se pueden determinar por las interacciones entre los mercados, para diversas mercancías. Su teoría se basó en supuestos restringidos -incluyendo la competencia perfecta- y no explicó cómo los precios se pueden determinar dada la existencia de mercancías del capital. No obstante su trabajo proporcionó los fundamentos para un trabajo más importante que amplió la teoría general del equilibrio, el de Kenneth Arrow y Gerard Debreu. Él también desarrolló la teoría marginal del valor con Guillermo Stanley Jevons y Carl Menger y ayudó a lanzar la escuela neoclásica en la economía. 
fica para retomar los principios epistemológicos y ontológicos que hemos heredado por esta cultura occidental (Monterroso, 2007), y por los políticos para impulsar nuevos modelos que nos permitan redireccionar el rumbo de las sociedades hacia una nueva racionalidad (Leff, 2007), o por qué no, a través de la ciencia post normal, en la que los científicos y profesionales tienen algo que decir, pero en la toma de decisiones se requiere incorporar a muchos otros actores sociales (Funtowicz, 1994).

\section{Antecedentes}

Aristóteles distinguió en su obra La Política, que el término oikonomia tiene dos sentidos: uno es el estudio del aprovisionamiento material del oikos o de la polis (casa) y el otro es el estudio de la formación de los precios con el deseo de ganar dinero, lo que propiamente es crematística (Martínez Allier, 2003). Sin embargo, a la postre, ha sido el orden el que más radicalmente ha manifestado el dislocamiento de la razón moderna, ya que ha existido un desprendimiento de la teoría sobre su referente ontológico, evolucionando a una racionalidad económica que ha transformado al ser humano actualmente en un homo economicus (Leff, 2004:135).

Para entender cómo surgió la ciencia económica debemos remontarnos al filósofo Thomas Hobbes $^{3}$, quien propuso que las sociedades, para ponerse de acuerdo, no estar en guerras y mantenerse en armonía unos a otros, crearon los Estados monárquicos, esto se hizo a través del Leviatán ${ }^{4}$ (Sorrell, 1986). Sin embargo,

3 Thomas Hobbes (5 de abril de 1588 - 4 de diciembre de 1679), fue un filósofo inglés, cuya obra Leviatán (1651) estableció la fundamentación de la mayor parte de la filosofía política occidental. Es el teórico por excelencia del absolutismo político. Hobbes es recordado por su obra sobre la filosofía política, aunque también contribuyó en una amplia gama de campos, incluyendo historia, geometría, teología, ética, filosofía general y ciencia política. Leviatán (en inglés Leviathan) es el libro más conocido del filósofo político inglés Thomas Hobbes, escrito en 1651. El nombre Leviathan hace referencia a un monstruo de la Biblia que posee un poder descomunal. En este libro,
Adam Smith ${ }^{5}$ argumentó que los miembros de la sociedad buscaron armonizarse a través del intercambio de riquezas por medio de la apertura del mercado y que una mano invisible era la que asignaba los recursos eficientemente, con lo que se lograba la prosperidad (Nadal, 2008).

Ahora bien, si el objeto de la economía es estudiar la asignación de recursos escasos a finalidades alternativas, actuales y futuras, asignación que se realiza mediante el sistema de precios, Karl Marx ${ }^{6}$ demostró lo contrario científicamente, porque adujo que tal asignación no existe, ya que, dentro del sistema económico capitalista una clase explota a otra, lo que publicó a través de la teoría del valor (plusvalía) y dedujo que la esencia del capitalismo es otra. Son las leyes económicas las que rigen el comportamiento de los individuos, dejando a un lado la ética porque hay objetos económicos como precios, monedas, mercancías, etc, (Marx, 1867).

Por ello, como la economía ha influenciado a las sociedades del mundo durante los últimos 250 años, los economistas neoclásicos que surgieron a finales de 1800 y principios de 1900, empezaron a hablar de un equilibrio social y compatibilizaciones de acciones sociales (Nadal, 2008), aunque se debe recordar que ya Marx había dicho que el mercado era un

Hobbes establece su doctrina de derecho moderno como la base de las sociedades y de los gobiernos legítimos

5 Adam Smith (5 de junio, 1723 - 17 de julio, 1790) fue un economista y filósofo escocés, uno de los máximos exponentes, si no el mayor, de la economía clásica. En 1776 publica: Ensayo sobre la naturaleza y las causas de la riqueza de las naciones, en la que sostiene que la riqueza procede del trabajo. El libro fue esencialmente un estudio acerca del proceso de creación y acumulación de la riqueza, tema ya abordado por los mercantilistas y fisiócratas, pero sin el carácter científico de la obra de Smith. Este trabajo obtuvo para él el título de fundador de la economía, porque fue el primer estudio completo y sistemático del tema.

6 Karl Heinrich Marx (Tréveris, Prusia, 5 de mayo de 1818 - Londres, 14 de marzo de 1883) fue filósofo, historiador, sociólogo, economista, escritor y pensador socialista alemán. Padre teórico del socialismo científico y del comunismo, junto a Friedrich Engels, es considerado una figura histórica clave para entender la sociedad y la política. 
error, ya que en el capitalismo una clase explota a otra y la armonía no existe, porque los agentes son egoístas (Marx, 1867).

Alfred Marshall7, quien recogiendo la teoría económica de los clásicos, con las aportaciones marginalistas de sus contemporáneos, realizó una síntesis en la que buscó y destacó las razones y requisitos de un eventual equilibrio parcial (Zaratiegui, 2002). Entonces surge Leon Walras como el primer economista que trata de demostrar matemáticamente la metáfora de la mano invisible, analizando y describiendo la Teoría General del Equilibrio para demostrar que efectivamente se podría alcanzar la armonía social. El problema es que a partir de entonces, los economistas no se dedicaron a estudiar a fondo las leyes del mercado, sino se encargaron mayormente en demostrar que la mano invisible sí funciona.

En el decenio 1930 la ortodoxia económica, basándose en los teóricos del equilibrio, sostuvo que el libre mercado dejado a sus propios mecanismos ordenaría espontáneamente a la sociedad de tal manera que optimizaría el bienestar humano (Napoleoni, 1972).

Sin embargo, aparece contra esta corriente económicamente dominante, John Maynard Keynes $^{8}$ quien mostró que era posible que las economías entraran en un equilibrio, pero de depresión, porque los diversos factores de producción no eran usados para alcanzar

$7 \quad$ Alfred Marshall, 1842-1924. Profesor de Economía Política en Cambridge, Reino Unido, es el fundador de la Escuela de Cambridge. Se le considera también precursor de la Economía del Bienestar, ya que su objetivo explícito en el análisis económico es encontrar una solución a los problemas sociales.

8 Economista británico, cuyas ideas tuvieron una fuerte repercusión en las teorías económicas y políticas modernas, así como también en las políticas fiscales de muchos gobiernos, generando el monetarismo. Es particularmente recordado por su aliento a una política de intervencionismo estatal, a través de la cual el Estado utilizaría medidas fiscales y monetarias con el objetivo de mitigar los efectos adversos de los periodos recesionarios de las fluctuaciones cíclicas o Crisis cíclicas de la actividad económica. Los economistas lo consideran uno de los principales fundadores de la macroeconomía moderna. configuraciones económicas óptimas. Por eso propuso que lo primero era asegurar el pleno empleo y abrió el camino de la idea para que el gobierno manejara la economía. En tal sentido, se atacó el esquema del libre mercado por la persistencia del desempleo, uso cerrado de los recursos disponibles y la tendencia a empresas monopólicas dentro de la economía (Eagly, 1968).

En muchos países el modelo keynesiano fue el motor económico para impulsar al capitalismo y se conoció como el modelo del Estado de Bienestar o del Estado de Providencia (Monterroso, 2007). Los mismos Estados Unidos de Norteamérica salieron adelante de la depresión económica en la que habían caído en la década de 1930, teniendo como principio fundamental que el Estado propiciara el pleno empleo. Pero en la década de 1970 aparecen Milton Friedman' y los "Chicago Boys", quienes retomaron la Teoría General del Equilibrio para proponer un nuevo modelo capitalista con los mismos principios del libre mercado. Surge entonces el Neoliberalismo y en 1973 se ensaya por primera vez la aplicación de este modelo en la República de Chile bajo la dictadura del fallecido General Augusto Pinochet, delegando al entonces ministro de hacienda, Enrique Iglesias, el desarrollo del mismo.

En 1982 se realiza el Concenso de Washington $^{10}$ y el expresidente de Estados Unidos, Ronald Reagan, conjuntamente con la Ex Primera Ministra de Inglaterra Margaret Thatcher (la

$9 \quad$ En 1976, fue galardonado con un Premio Nobel de Economía por sus logros en los campos de análisis de consumo, historia y teoría monetaria y por su demostración de la complejidad de la política de estabilización.

10 El "Consenso de Washington" debe su nombre al economista inglés John Williamson, quien a fines de la década de los 80 se refirió así a los temas de ajuste estructural que formaron parte de los programas del Banco Mundial y del Banco Interamericano de Desarrollo, entre otras instituciones, en la época del re-enfoque económico que siguió a la crisis de la deuda desatada a principios de la década. Algunos se refieren a este acuerdo como la "Agenda de Washington", otros como la "Convergencia de Washington" y unos pocos la denominan "Agenda Neoliberal". 
dama de hierro), acuerdan impulsar el nuevo modelo de libre mercado por todo el mundo (Larraín, 2004).

A partir de entonces, y debido a la caída de la cortina de hierro y consecuente desintegración de la Unión Soviética, en 1990, al desaparecer el sistema socialista (Bonilla, 2007), el libre mercado fue adoptado por prácticamente todo el mundo, predominando el modelo neoliberal como impulsor de la economía, dando paso a la "Globalización", teniendo impactos adversos sobre las sociedades económicamente más desiguales y sobre el ambiente (Ramírez, 2008).

\section{Teoría general del equilibrio}

Como ya se ha mencionado, desde el punto de vista más estricto la economía debería de armonizar a las sociedades asignando los recursos bajo el principio de competencia perfecta, de tal manera que el mercado permitiría compatibilizar planes individuales de agentes egoístas y alcanzar el óptimo (prosperidad). Este equilibrio sólo se logra con precios justos, a lo que se denomina $P^{\star}$ (precios en equilibrio). Esto es un estado deseable en el que se encuentran los precios y las cantidades físicas de una economía representados como un triple de vectores $\left(X^{\star}, Y^{*}, P^{\star}\right)$, siendo $X^{\star}$ las mercancías en cantidades demandadas con precios justos, $Y^{*}$ las mercancías en cantidades ofrecidas con precios justos y $\mathrm{P}^{\star}$ es la oferta y la demanda en equilibrio.

Para Smith (1776), el mercado asigna eficientemente los recursos, lo cual trató de explicar a través de la metáfora de la mano invisible. Sin embargo, no lo demostró y únicamente dijo que había algo que no se podía explicar, pero que regulaba el mercado, y es lo que conocemos hoy como la ley de la oferta y la demanda. Esta ley nos dice que cuando los precios suben la demanda de un producto baja y por el contrario cuando la demanda de un bien o servicio es alto, los precios del mismo tienden a bajar. Desde entonces, para los economistas lo más importante no fue estudiar las leyes del mercado, sino demostrar que el equilibrio se puede alcanzar para obtener un estado de bienestar deseable. Wilfredo Pareto ${ }^{11}$ propuso un teorema que los economistas conocen como Óptimo de Pareto, el cual se basa en lograr que una persona mejore sin dañar a otra.

La dominancia de Pareto indica que:

Dado un vector

$$
\mathbf{u}=\left(u_{1}, \cdots, u_{k}\right)
$$

se dice que domina a otro vector

$$
\mathbf{v}=\left(v_{1}, \cdots, v_{k}\right)
$$

si y sólo si:

$\forall i \in\{1, \cdots, k\}, u_{i} \leq v_{i} \quad y \exists i_{0} \in\{1, \cdots, k\} \mid u_{i_{0}}<v_{i_{0}}$

Lo anterior sugiere que una solución $X^{\star}$ es $\mathrm{Pa}$ reto-óptima si y sólo si no existe otro vector $X$ tal que $\mathbf{v}=f(\mathbf{x})=\left(v_{1}, \ldots ., v_{k}\right)$ domine a $\mathbf{u}=f\left(\mathbf{x}^{*}\right)=\left(u_{1}, \ldots ., u_{k}\right)$

Por lo tanto, el punto $\mathrm{X}^{\star}$ es un óptimo de $\mathrm{Pa}$ reto si no existe un vector $X$ que haga mejorar alguno de los objetivos -respecto a los valores obtenidos para $X^{*}$ - sin que empeore de forma simultánea alguno de los otros. En general, la solución en el sentido de Pareto al problema de optimización multiobjetivo no será única: la solución estará formada por el conjunto de todos los vectores no dominados, a los que se conoce con el nombre de conjunto de no dominados o frente de Pareto.

El sentido del Óptimo de Pareto es demostrar que sólo en el equilibrio hay eficiencia y que el mercado cumple con esta función pa-

11 Wilfredo Federico Damaso Pareto (15 de julio de 1848, París - 19 de agosto de 1923, Ginebra) fue un sociólogo, economista y filósofo italiano que realizó importantes contribuciones al estudio de la economía y de la sociología, especialmente en el campo de la distribución de la riqueza y el análisis de las elecciones individuales. Fue el creador del concepto eficiencia de Pareto y contribuyó con ideas como la de la curva de indiferencia al desarrollo de la microeconomía. 
sando de un $\mathrm{P}$ (precio sin equilibrio) a un $\mathrm{P}$ * (precio en equilibrio). El economista Kenneth Arrow $^{12}$ se dedicó a analizar y demostrar la Teoría General del Equilibrio, proponiendo que para que exista una formación de precios en equilibrio, el proceso debe ser dinámico, con demostración matemática (es a lo que se le conoce como axiomatización), y el modelo a aplicarse debe ser sin intervención del Estado, descentralizado, con objetivo doble y los individuos deben buscar maximizar la función (Nadal, 2007).

Sin embargo, las contradicciones e inconsistencias cuando en la aplicación del modelo de mercados abiertos se espera que la tasa de cambio fluya libremente para mantener el equilibrio en el balance de las transacciones, pero, en la práctica, esta tasa cambia lo menos posible para evitar la inflación y así garantizar los riesgos que correría una eventual inversión extranjera. Además, la tasa de interés a nivel doméstico también se espera que fluctúe para mantener el equilibrio del capital en el mercado, no obstante, es usada para regular el suministro de dinero, la tasa de inflación y el nivel de la actividad económica.

Por su parte, la liberación de mercados a menudo guía a los flujos del capital extranjero, pero estos flujos pueden acrecentar la capacidad financiera para importar más rápidamente que la capacidad productiva exportadora. Otra contradicción que se presenta es que la desrregulación de las instituciones financieras es necesaria para incrementar los ahorros domésticos y de inversión, pero la misma desrregulación también incrementa las oportunidades para la inversión especulativa y la

12 Kenneth Joseph Arrow, economista estadounidense que nació el 23 de agosto de 1921 en Nueva York. Estudió en el City College de esa ciudad y, luego de terminar su maestría en 1941, obtuvo su doctorado en la Universidad de Columbia en 1951. Se hizo acreedor al Premio Nobel de Economía en 1972, al lado de John Richard Hicks. Sus principales contribuciones han sido en el campo de la teoría de la decisión, especialmente su teorema de imposibilidad de la democracia y el análisis del equilibrio general en microeconomía. salida del capital doméstico hacia los mercados exteriores.

Por último, una de las más grandes contradicciones es el hecho que el desarrollo de un país bajo los modelos de economías abiertas requiere la promoción de un sector exportador exitoso, un proceso que históricamente ha requerido participación del Estado. Pero la lógica del modelo de economías abiertas se inclina hacia la reducción y el debilitamiento de la capacidad del Estado para intervenir en el mismo mercado.

\section{Funcionalidad teórica del modelo de mercados abiertos}

Como ya se ha recalcado, el Modelo de Mercados Abiertos se basa en la Teoría General del Equilibrio la cual busca pasar de precios $\sin$ equilibrio a precios en equilibrio $\left(P \rightarrow P^{\star}\right)$. Para que esto sea demostrado, se requieren teoremas axiomatizados ${ }^{13}$, tales como el de la Estabilidad Global y el de la Estabilidad Local (Nadal, 2008). En un modelo típico de equilibrio general, los precios que prevalecen son aquellos que compatibilizan las demandas de los diferentes consumidores con las distintas mercancías, cuando las condiciones generales de la economía se estabilizan.

A partir de ello surge la pregunta sobre cuál ha sido el proceso mediante el cual la economía supuestamente ha llegado a ese estatus de equilibrio. La situación se complica cuando para ser demostrado a través de axiomas se necesita de supuestos. Para pasar de $P \rightarrow P^{\star}$ son necesarios los supuestos de Sustitutabilidad Bruta (SB) y el Axioma Débil de Preferencias Reveladas (ADPR) a nivel de mercado.

La sustituibilidad explicaría que, entre bienes dada cualquier combinación inicial, digamos a unidades del bien $x$ y $b$ unidades del bien $y$, un consumidor casi siembre estaría dispuesto a renunciar a una pequeña

13 Al axiomatizarse un teorema toma un carácter irrefutable. 
cantidad del bien $\boldsymbol{x}$, si se le ofrece como compensación una cantidad adicional del bien y que sea lo suficientemente grande (cantidad variable en función del punto de partida y de las preferencias de cada cual). Así, si hablamos de dos mercancías, existiría una "relación subjetiva de intercambio", que habría de comportarse con la relación objetiva de intercambio dada el precio relativo de mercado entre los bienes; sólo cuando ambas coincidiesen el consumidor estaría en el óptimo. (...) Nótese que la palabra "necesidades" es ajena al planteamiento anterior. Necesidades, deseos, caprichos...están al mismo nivel, de manera que incluso se hace imposible explicar -más allá de la explicación tautológica de que las preferencias o la función de utilidad así lo determinan- la regularidad empírica del consumo más universalmente constatada, y que se conoce como "ley de Engel", según la cual los pobres gastan en alimentos un mayor porcentaje de su renta que los ricos (Martínez Allier, 2003).

El Axioma Débil de Preferencias Reveladas (ADPR) a nivel de mercado, indica que cuando existen dos canastas $x$, un consumidor al no poder alcanzar $x_{1}$ toma $x_{2}$. En teoría esto po- dría representarse matemáticamente, pero en la práctica a veces no es tan fácil. El aumento en el consumo de cualquier bien supliría un mismo y único objetivo: aportar utilidad (o, lo que es lo mismo, permitir situarse en un lugar más avanzado dentro del orden de preferencias). Las combinaciones de bienes que proporcionan mayor o menor utilidad serían una cuestión subjetiva, y totalmente abierta, puesto que "sobre gustos no hay nada escrito" (Martínez Allier, 2003).

Las investigaciones realizadas sobre los modelos matemáticos que tratan de explicar el equilibrio general, sugieren que a pesar que los teoremas han demostrado (parcialmente, porque se necesita de supuestos) que existe una competencia perfecta, en la realidad esto no se cumple. Para que resulte, nadie tendría que influir sobre los precios. Como también ya hemos mencionado, Adam Smith en 1775 sugirió que hay "una mano invisible" que asigna los recursos eficientemente, pero es Irving Fisher ${ }^{14}$ quien trata de estudiar esta famosa "mano invisible" a la cual denomina auctionner o subastador. De acuerdo con Nadal (2008), se puede ilustrar el funcionamiento del subastador para formar precios en equilibrio a través de un modelo denominado tâtonnement ${ }^{15}$, el cual lleva el orden de la Figura 1:

14 Irving Fisher es un economista estadounidense que contribuyó a difundir las ideas económicas neoclásicas en Estados Unidos y dio formulaciones muy modernas para su época: fue el inventor de los índices económicos y un pionero de la econometría.

15 De la lengua francesa tanteo. 


\section{Figura 1}

Formación de precios en equilibrio a través del método tâtonnement

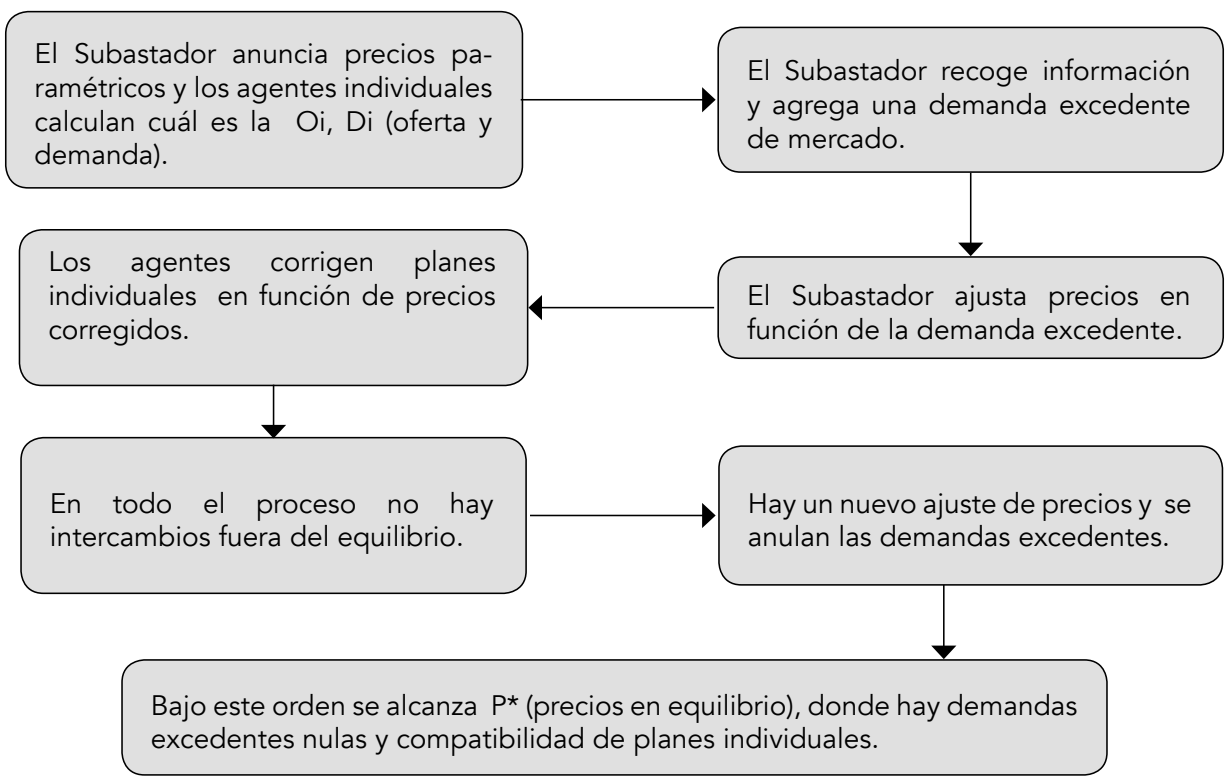

Fuente: Elaboración propia

Como podemos darnos cuenta, se alcanza el equilibrio donde hay demandas excedentes nulas y compatibilidad de planes individuales. Sin embargo, no es tan sencillo como parece, ya que se necesita de algunos supuestos tales como: la Ley de Walras, que nos indica que no se puede consumir más de lo que tenemos de presupuesto; la Ley del Subastador, que es equivalente a la ley de la oferta y demanda o mano invisible que regula los precios; ley de Continuidad y Ley de Homogeneidad. Además, recordemos los otros supuestos de Sustitutabilidad bruta (SB) y el Axioma Débil de Preferencias Reveladas (ADPR) a nivel de mercado. A continuación se presentan los modelos estructurales de los supuestos en mención en la Figura 2:

Figura 2

Supuestos estructurales de la estabilidad global con tâtonnement

$$
\begin{array}{ll}
\text { Continuidad } & \text { Si } \lim _{n \rightarrow \infty} x_{n}=x 0 \Rightarrow \lim _{n \rightarrow \infty} f\left(x_{n}\right)=f\left(x_{0}\right) \\
\text { Homogeneidad } & z_{i}(p)=z_{i}(\alpha p), \alpha>0, \alpha \in R \\
\text { Ley de Walras } & \sum_{i=1}^{n} p_{i} \cdot z_{i}(p)=0 \\
\text { Regla del subastador } \frac{d p_{i}}{d t}=G_{i}\left[z_{i}(p)\right] \quad \begin{array}{l}
\text { En donde G } \\
\text { conserva el } \\
\text { signo de z }
\end{array}
\end{array}
$$

Fuente: Nadal, 2008. 
De acuerdo con estos supuestos, surgen algunas interrogantes que se deben plantear: ¿Cómo están articulados todos estos supuestos? ¿Bajo qué condiciones son consistentes entre sí? Más importante: ¿son consistentes con el objeto de la teoría? Si se aceptan todos los supuestos: ¿se alcanzan los resultados deseados? Es probable que se demuestre la estabilidad global con tâtonnement a través del Lemma $^{16} \sum p^{\star} i \cdot z i(p)>0$. El problema es que se debe mantener a los otros supuestos de Sustitutabilidad bruta (SB) y el Axioma Débil de Preferencias Reveladas (ADPR) a nivel de mercado, los cuales son absurdos porque en el proceso de formación de precios los agentes son individuales, además hay una presencia de un subastador y se tienen relaciones monetarias y precios relativos.

Al no ser satisfactorio el resultado de los teoremas hasta entonces desarrollados, en 1958 Herbert Eli Scarf ${ }^{17}$ publica su célebre contraejemplo "La conjetura de Arrow" y al poco tiempo se plantea otro modelo denominado non-tâtonnement en 1974, propuesto por Sonnenschein, Mantel y Debreu ${ }^{18}$ el cual también presenta sus implicaciones sobre el subastador y el sistema de precios e intercambios sólo en el equilibrio. Para entonces, Negishi, Hahn y Fisher proponen otros supuestos, aunque se retiene al subastador, se reemplaza la prohibición de realizar intercambios fuera del equilibrio por el supuesto de los mercados ordenados. Esto se logra por la Regla de Hahn, donde: $\mathrm{Zhi} \cdot \mathrm{Zi}>0$ para todo $\mathrm{h}, \mathrm{i}$; y si $\mathrm{Zi}=0$,

Zhi $=0$ para todo $\mathrm{h}$, i (Zhi $\equiv$ demanda de $\mathrm{i}$ por agente $\mathrm{h} ; \mathrm{Zi} \equiv$ demanda excedente de i) (Nadal, 2008).

16 Modelo matemático para comprobar una declaración.

17 Herbert Eli "Herb" Scarf, es un economista de la Universidad de Yale. Es miembro de la Academia Americana de Artes y Ciencias. Obtuvo su Ph. D. en Princeton en 1954. Fue pionero en el uso de algoritmos para explicar el equilibrio general a través de modelos aplicados.

18 Gerard Debreu (4 de julio de 1921-31 de diciembre de 2004) fue un economista nacido en Francia, pero nacionalizado estadounidense, que ganó el Premio Nobel de Economía en 1983.
Ahora se llevan a cabo los intercambios fuera del equilibrio. La regla de Hahn permite que las utilidades objetivo se vayan reduciendo. Se utiliza un teorema de Lyapunov: el equilibrio y las utilidades esperadas objetivo se anulan. Se terminaron las oportunidades de intercambios favorables. El modelo non-tâtonnement es interesante porque no requiere $\mathrm{SB}$, ni ADPR, únicamentes mantiene el subastador, aunque se pierde la unicidad del equilibrio, y algo más importante: sigue siendo un modelo no monetario. Pero, entonces, ¿cómo se llevan a cabo los intercambios?

Supongamos que se alcanza (por un modelo de tâtonnement) un Equilibrio General Comercial $\left(x^{*}, y^{*}, p^{\star}\right)$. Ahora el subastador anuncia que se trata de precios de equilibrio y que se pueden llevar a cabo los intercambios. ¿Está resuelto el problema del mercado? ¿Se pueden vaciar los mercados y los agentes individuales pueden realizar sus planes? Si el modelo de non-tâtonnement tiene menos restricciones que el modelo de tâtonnement ¿por qué no ha tenido tanto éxito? Una primera razón es porque se pierde la unicidad. La segunda es porque al modelo de non-tâtonnement no se le pone en presencia de problemas más profundos, pero en especial, porque en ausencia de moneda, no se cumple la regla de los mercados ordenados (regla de Hahn).

Por lo tanto, los problemas en la teoría de formación de precios indican que nos encontraríamos con una situación complicada con el concepto de moneda. La moneda (fiduciaria) y las mercancías guardan una posición asimétrica en la teoría del valor: Las mercancías tienen valor positivo (poder de compra) en la medida en que tienen utilidad (valor de uso): Con la moneda (fiduciaria) es lo contrario: tiene utilidad en la medida en que tiene poder de compra (precio positivo), pero siempre hay que suponer que pm (moneda) > 0 . Entonces ¿Cómo justificar el precio positivo en todo momento de un bien que no tiene utilidad intrínseca? 
Notemos que los modelos más refinados y sofisticados de la Teoría General del Equilibrio para analizar al mercado no usan la moneda tanto fiduciaria como la de mercancía por su asimetría. La fiduciaria es un objeto económico que no tiene valor de uso propio y es útil cuando tiene el poder de comprar, por lo que siempre debe ser positivo, por eso todo el mundo la acepta. La mercancía tiene el valor de uso propio y utilidad propia, por eso Smith (1776), dijo que la riqueza no estaba en el oro, si no en el intercambio de la producción de bienes y servicios, aunque, esta es actualmente para muchos economistas una deficiente teoría monetaria. Total, es tan contradictorio todo esto, porque la teoría económica nace haciendo a un lado a la moneda a pesar que en la realidad si contamos con ella, y por el otro lado, no todos necesitaríamos sólo de una mercancía en particular (peras y tomates), sino de otras mercancías para satisfacer nuestras necesidades (Nadal, 2008).

En su obra "Ecuaciones e incógnitas", Walras había propuesto una solución al equilibrio, pero no fue suficiente. En 1950, John Nash ${ }^{19}$ utiliza un nuevo instrumento matemático para juegos cooperativos de $n$ personas. El Teorema de Punto Fijo para funciones univaluadas (Brouwer), en donde: sea $S$ un subconjunto compacto, convexo, no vacío de $R_{n}$ y sea f una función univaluada continua de $\mathrm{S}$ en sí mismo, entonces, existe una $p^{\star}$ en $S$ tal que $p^{\star}=f\left(p^{\star}\right)$. También encontramos el Teorema de Punto Fijo para correspondencias semi continuas superiormente (Kakutani), el cual nos indica que,

19 John Forbes Nash Jr. (Bluefield, Virginia Occidental, 13 de junio de 1928) es un matemático estadounidense. Recibió el Premio Nobel de Economía de 1994 por sus aportes a la teoría de juegos y los procesos de negociación, junto con Reinhard Selten y John Harsanyi. Sus teorías han influido en las negociaciones comerciales globales, en los avances en biología evolutiva, en las relaciones laborales nacionales y en problemas de Toma de Decisiones. Se ha rodado una película titulada A beautiful mind (Una mente maraviIlosa o Una mente brillante), estrenada en 2001, interpretada por Russell Crowe y dirigida por Ron Howard. Ganadora de cuatro premios Oscar, incluyendo la categoría de mejor película, la película se basa en la biografía de Nash escrita por Sylvia Nasar (1999), centrándose principalmente en la esquizofrenia que sufría Nash. sea $\mathrm{S}$ un subconjunto compacto, convexo y no vacío de Rn y sea $f$ una correspondencia semi-contínua superiormente de $\mathrm{S}$ en sí mismo tal que para todo $p S$, el conjunto $f(p)$ es no vacío y convexo, entonces, existe una $p^{\star} S$ tal que $p^{\star} € f\left(p^{\star}\right)$.

Como es de suponer, todo lo anterior debe ser demostrado, y aquí surge nuevamente otro problema, porque el mapeo en la teoría de Punto Fijo debe tener un sentido económico, y el problema de la integración de la moneda aun permanece sin solución, por lo tanto no hay una demostración sobre el proceso que permite la formación de los precios de equilibrio.

\section{Teoría economía y medio ambiente}

Las contradicciones de la teoría económica relacionadas al ambiente no son la excepción. Los mismos economistas que más admiran al mercado admiten que, cuando se impacta al ambiente, sí se justifica la intervención estatal. El problema ambiental es que no existe un mercado de "bienes ambientales", por lo que Ronald Coase $^{20}$ propone en su artículo "The Problem of Social Cost", que la solución sería crear un mercado allá donde no existe. Este economista ha sido autor de unos pocos artículos que han sido considerados lo suficientemente importantes como para merecer el premio Nobel de Economía en 1991. La economía ambiental está basada fundamentalmente en conceptos como "derechos de propiedad" sobre el ambiente y "costes de transacción".

20 Ronald Howard Coase nació en Willensden Reino Unido ( 29 de diciembre, 1910), economista británico y profesor emérito en la Universidad de Chicago. Recibió el Premio Nobel de Economía en 1991 por el descubrimiento y aclaración del significado de los costes de transacción y derechos de propiedad para la estructura institucional y el funcionamiento de la economía. Emigró a Estados Unidos, trabajando en la Universidad de Buffalo, en el Center for Advanced Studys in the Behavioral Sciences, en la Universidad de Virginia y, desde 1964, en la Universidad de Chicago en la escuela de leyes. Fue editor de la revista "Law and Economics" de 1964 a 1982. 
Coase plantea diversos ejemplos de conflicto de intereses entre dos agentes económicos (algunos de ellos, casos reales que provocaron demandas judiciales). Uno es el de un confitero que usa un instrumento de trabajo que produce ruidos y vibraciones que molestan al médico que tiene la consulta contigua a su taller. El argumento de Coase es que es inadecuado pensar en el confitero como el culpable que o bien ha de cesar de provocar molestias, o bien ha de compensar necesariamente al médico. Según él, el problema es recíproco, ya que las dos actividades -la del médico y la del confitero- son igualmente legítimas y, de la misma forma que obligar al médico a trasladar su consulta, o a insonorizarla, o a los pacientes a soportar los ruidos, o acudir a otro médico, comporta unos costes -"coste de oportunidad" o beneficio que deja de percibirse por el confitero o por sus clientes- impedir al confitero utilizar una tecnología que reduce los costes de su actividad (Martínez Allier, 2003).

Para los economistas neoclásicos, los impactos que produce la economía al ambiente no son más que fallas del mercado a lo cual denominan "externalidades". Coase, preocupado por la "eficiencia económica", dice que lo importante es comparar lo que se pierde al dejar de utilizar una tecnología con el que se pierde por las molestias que provoca, por lo que argumenta que la solución a las fallas del mercado es "internalizar" dichas fallas, valorando económicamente a la contaminación y dejar que el mercado solucione los problemas ambientales. El teorema de Coase ${ }^{21}$, formula que si las par-

21 No es el premio Nobel de Economía Ronald Harry Coase, sino George Stigler, quien establece la expresión "teorema de Coase", y se acostumbra a formular más o menos así: "en ausencia de costes de transacción, el resultado económico, por lo que se refiere a la asignación de recursos, es siempre el mismo (y eficiente), con independencia de cómo se distribuyan los derechos iniciales, siempre que éstos estén claramente definidos". Los argumentos del propio Coase son los siguientes: ante presencia de determinadas externalidades (que Coase prefiere denominar efectos externos) siempre será posible la con- tes privadas y públicas que están implicadas en la existencia de unas externalidades pueden negociar sin ningún costo sobre la asignación de los recursos, pueden resolver por sí solas el problema mismo. Dicho de otra forma, si no existen los llamados "costes de transacción", son irrelevantes las reglas de responsabilidad civil para garantizar una asignación óptima de los recursos, en el sentido de la eficiencia social representada por la llamada "Eficiencia de Pareto" (Coase, 1960).

Sin embargo, el mismo Coase advirtió enfáticamente que su argumentación "no implica que cuando los costes de transacción son positivos, las actividades gubernamentales (tales como intervenciones estatales, la regulación a los impuestos, incluidos los subsidios) no produzcan mejores resultados que el basarse en negociaciones entre individuos a través del mercado. Mi conclusión: estudiemos el mundo de costes de transacción positivos (Coase, 1994).

Por lo tanto, podemos demostrar la falsedad de esta teoría a través de dos aspectos: el primero es que cuando una persona se ve afectada por las acciones de otra, la "disposición a pagar" para evitar la molestia, no coincide con la "disposición a aceptar una compensación" para soportarla. Por ejemplo, si a una persona se le pregunta cuánto dinero pagaría para evitar que se construya una presa hidroeléctrica que inundará su casa, o se le pregunta, en cambio, cuánto aceptaría para dar su aprobación al proyecto, es posible que la persona no acepte la primera pregunta y responda que tiene un derecho por el que no tiene que pagar (así de sencillo). La segunda, es una in-

secución de una externalidad óptima (lo cual no implica necesariamente la desaparición total de la misma) y de un máximo nivel de bienestar. Esto se logrará a través de la negociación. Para ello es necesario que los derechos de propiedad de las distintas partes estén bien asignados y pueden defenderse. Otra condición es que el sistema de precios funcione sin costes y no existan efectos renta, en el marco del equilibrio general. Es una crítica a la vía tradicional intervensionista de Pigou, que ha mostrado no ser todo lo eficiente que se esperaba, a pesar de su amplia aceptación en la literatura y teoría económica. 
consistencia muy grosera, porque Coase en su artículo tiene la preocupación de referirse sobre todo a casos en que únicamente hay dos agentes económicos afectados. Sin embargo, la mayor parte de los problemas ambientales relevantes afectan a una colectividad e incluso, a veces, a toda la humanidad y a las generaciones futuras. Por lo tanto, el mercado no da solución por sí solo a los problemas ambientales, por muy bien delimitados que estén los derechos de propiedad (Martínez Allier, 2003).

En 1970, el Club de Roma, una asociación privada compuesta por empresarios, científicos y políticos, encargó a un grupo de investigadores del Instituto Tecnológico de Massachussets, (MIT por sus siglas en Inglés), la realización de un estudio sobre las tendencias y los problemas económicos que amenazan a la sociedad global. Los resultados fueron publicados bajo el título "Los Límites del Crecimiento". En ese estudio se utilizaron las técnicas de análisis de dinámica de sistemas más avanzadas del momento. En primer lugar se recopilaron datos sobre la evolución que habían tenido en los primeros setenta años del siglo XX un conjunto de variables: la población, la producción industrial y agrícola, la contaminación, las reservas conocidas de algunos minerales. Diseñaron fórmulas que relacionaban esas variables entre sí -la producción industrial con las existencias de recursos naturales, la contaminación con la producción industrial, la producción agrícola con la contaminación, la población con la producción agrícola, etc.y comprobaron que esas ecuaciones sirvieran para describir con fidelidad las relaciones entre los datos conocidos que habían recopilado. Finalmente introdujeron el sistema completo en un ordenador y le pidieron que calculase los valores futuros de esas variables. Las perspectivas resultaron muy negativas. Como consecuencia de la disminución de los recursos naturales, después del año 2000 se produciría una grave crisis en las producciones industrial y agrícola que invertirían el sentido de su evolución. Con algún retardo la población al- canzaría un máximo histórico a partir del cual disminuiría rápidamente. Hacia el año 2100 se estaría alcanzando un estado estacionario con producciones industrial y agrícola per cápita muy inferiores a las existentes al principio del siglo XX y con la población humana en decadencia (Meadows, 1972).

No obstante, Simon Kuznets ${ }^{22}$ sugirió que el crecimiento económico no era del todo malo, ya que este aseguraría que se tome más cuidado sobre el ambiente porque a medida que se alcancen los recursos económicos necesarios, se podría invertir más en el ambiente para conservarlo y en ese sentido se alcanzaría "la eficiencia". Para Kuznets el crecimiento económico no solo se convertía en un problema, sino sería la solución al tema ambiental (Nadal, 2008).

\section{Figura 3}

\section{Curva Ambiental de Kuznets (EKC) ${ }^{23}$}

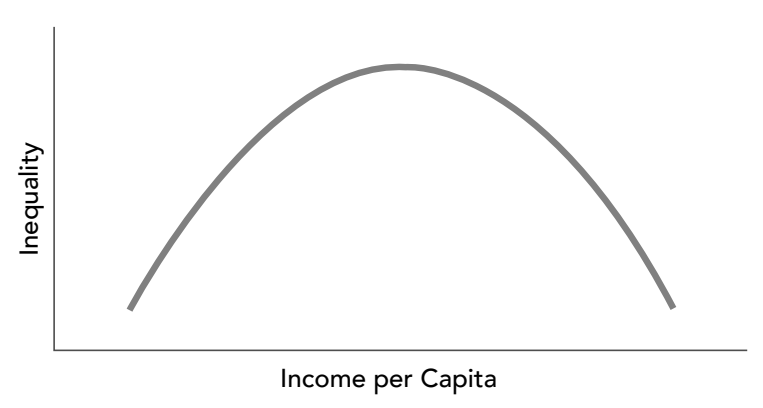

22 Simon Kuznets fue un economista ruso-estadounidense, nacido en Rusia en 1901. De padres judíos, inició sus estudios universitarios en su país natal, pero en 1922 se mudó a Estados Unidos terminando sus estudios en la Universidad Columbia. Trabajó de profesor en la Warthon School de la Universidad de Pensilvania, en la Universidad Johns Hopkins y en la Universidad Harvard. En una hipótesis relacionó el crecimiento económico y la distribución del ingreso. Según ésta, el crecimiento basta para reducir la desigualdad, aunque ésta también se asocia a los comienzos del crecimiento, cuando existe la necesidad de realizar grandes inversiones en infraestructura y en bienes de capital. Luego la generación de empleo y el aumento de la productividad conducirían a salarios más elevados y a una mejor distribución del ingreso.

23 La curva de Kuznets es la representación gráfica de la teoría de Simon Kuznets, donde crece la desigualdad económica a través del tiempo mientras un país desarrolla, pero luego al llegar a un ingreso per cápita óptimo la desigualdad decrece. 
Sin embargo, esta teoría también depende de algunos supuestos, ya que se debe de tener en un país cambios estructurales y técnicos en la economía, porque sólo de esa manera se alcanzaría el umbral del PIB per capita necesario, el cual, para países latinoamericanos en promedio tendría que ser de $\$ 12,000.00$. Sin embargo, para alcanzar dicho umbral, la demanda de recursos naturales sería sumamente grande, por lo que todo resultaría en una sustentabilidad débil (Martínez Allier, 2006).

El argumento de la curva ambiental de Kuznets está basado sobre el desarrollo industrial y la economía. El interés es dar peso para limpiar al ambiente a través de la reducción de la contaminación industrial. Esta tendencia parabólica sucede a nivel de muchos factores contaminantes como el dióxido de azufre, óxido nitroso, DDT, productos órgano clorados y fosforados, aguas servidas y otros químicos liberados directamente sobre el aire y agua (Grossman, 1993).

El problema de la curva ambiental de Kuznets es que no toma en cuenta que el medio ambiente es un vector multidimensional y para alcanzar el umbral económico requerido, supone un deterioro ambiental acumulativo y en esto no hay irreversibilidades. Por ejemplo, al extinguirse una especie endémica de un ecosistema producto de las actividades económicas ninguna cantidad monetaria alcanzaría para volver a la vida a la especie extinta. El PIB per cápita no tiene correlación con la intensidad de emisiones de gases de efecto invernadero en todos los países del mundo. Por último, no se toma en cuenta el desplazamiento de costos ambientales y los efectos acumulativos e interdependencias de la posibilidad de un colapso ambiental.

\section{Efectos del modelo de mercados abiertos en América Latina}

Ramírez (2008), muestra de forma sistematizada los efectos negativos que se han tenido sobre las sociedades latinoamericanas por la aplicación del modelo de Mercados Abiertos
- neoliberalismo, producto de las inconsistencias y contradicciones de los fundamentos teóricos del equilibrio general:

- Se ha creado una férrea política de austeridad monetaria en aras de mantener en el mínimo la tasa de inflación, lo que podría traducirse en una disminución abrupta e inhumana de los gastos sociales del Estado.

- Se ha organizado la economía en función de una política netamente exportadora (aquí por supuesto, se hace total abstracción del hecho simple de que el mercado, aun el ideal, pone límites naturales como resultado de la ley de oferta y demanda y que además las exportaciones tercermundistas tienen que competir con las producciones de los países industrializados, que controlan los mercados y de hecho son mercados en sí mismos).

- Se ha aumentado de forma alarmante la especulación financiera.

- Se ha consolidado como sector hegemónico en la región el capital financiero nacional e internacional, unido a la industria más concentrada y selectiva, orientada a la exportación y ampliamente integrada a la economía mundial, y a la vieja oligarquía (latifundista e intermediaria), pero modernizada y diversificada.

- La ausencia de inversiones en sectores clave provocó un mayor atraso económico; de otra parte, sistematizó la volatilización de los capitales y su fuga constante. En 1995, el monto global de los depósitos de las clases dominantes latinoamericanas y caribeñas fugados hacia bancos de fuera de la región (en particular, a los de origen estadounidense) ascendió a 366 millones de dólares: cerca del 50\% de la deuda externa desembolsada en 1998.

- El modelo económico neoliberal ha provocado fuertes crisis económicas como la de 
México en 1994, conocida como Efecto Tequila, o en Argentina, que privatizó al país para recoger como resultado el más destacado desempleo conocido en la historia de los países latinoamericanos.

- Se ha aumentado aceleradamente la deuda. Por ejemplo, la deuda externa regional ascendió de cerca de 290000 millones de dólares en 1981 a casi 540000 millones en 1994. Entre 1982 y 1996 el continente pagó por los servicios de esa deuda aproximadamente 706 millones de dólares y en 1998 la deuda desembolsada ascendió a 740905 millones de dólares, más del triple de su monto en 1980.

- Se ha revelado también un mal crónico: el déficit de cuenta corriente que en 1997 fue de 64000 millones y en 1998 creció hasta 84000 millones.

- Las privatizaciones de empresas estatales y la estrategia exportadora establecidas por el modelo neoliberal privilegiaron ante todo al capital financiero, a la burguesía más concentrada y a la oligarquía agroexportadora modernizada. La hegemonía de estos sectores se definió en la medida en que pudieron acceder al mercado externo, tanto para colocar sus productos como para financiarse, lo cual dejó claro que el Estado no era una institución débil frente al mercado, como se pretendía hacer ver, sino que los sectores más débiles que anteriormente gozaban de cierta bonanza proveniente del presupuesto estatal, fueron definitivamente expulsados de ese vínculo y devueltos a su sitio, a su rol de mercancía barata que reproduce el ciclo del capital. Partiendo de su supuesta ineficiencia, se expulsó al Estado de la producción de bienes y servicios, se liquidó cualquier vínculo de este con la distribución de los ingresos, los cuales se concentraron legitimados por elecciones y demás procesos formales en un grupo social muy reducido.
Se desrreguló por completo el sistema de contratación de la fuerza laboral, las jubilaciones y todo el aparato de asistencia social. Se pasó a un régimen económico de exportación masiva de producción primaria y de ciertas ramas industriales, que desligó la producción de la satisfacción de las necesidades de la población.

- Se ha aumentado la anarquía en la gestión económica por la ausencia de entidades capaces de controlar las inversiones.

- Se ha fortalecido la presencia de las empresas transnacionales en detrimento de las empresas públicas.

- Como resultado de todo el discurso neoliberal los capitalistas locales y sus socios metropolitanos han obtenido varias ventajas: primero, reforzaron de manera considerable su predominio económico, reduciendo drásticamente el control público de los recursos nacionales y facilitando el accionar del sector privado. Segundo, algo muy importante para el gran capital financiero internacional y del cual se habla muy poco: garantizaron el pago de la deuda externa, destinando a ella todos los efectos recursos y propiedades de carácter público otrora intocables; tercero, modificaron a su favor, y de manera decisiva, la correlación de fuerzas entre el mercado y el Estado, condicionando de este modo los grados de libertad que pudiera tener algún futuro gobierno animado por una vocación reformista o transformadora.

- A nivel social, la consecuencia máxima y otra de las grandes victorias que obtuvieron los propulsores del neoliberalismo, de acuerdo a los objetivos que se plantearon, fue la creación de un sistema que exacerbaba el individualismo, que privilegiaba y estimulaba la confrontación por encima de la cooperación. 
- El eufemismo24 de la flexibilización de los mercados laborales ha creado en América Latina una situación caótica con la innovación que introdujeron las maquiladoras, mientras que para el capital transnacional esto constituyó el mejor de los negocios, pues se rentaron terrenos poco valorizados, se instalaron industrias desmontables y contrataron fuerza de trabajo con salarios prácticamente simbólicos. Todas estas acciones con la intención de elevar la tasa de beneficio del capital, disminuyendo el costo de producción.

- Se ha producido un deterioro físico e intelectual de la fuerza de trabajo, que se fue haciendo cada vez menos apta para la actividad transformativa.

- Según diversos trabajos elaborados por la CEPAL, en 1960 un 51\% de personas vivía por debajo de la línea de pobreza en América Latina, lo que equivalía a unos 110 millones de personas. En 1970 esta proporción descendió sensiblemente a un $40 \%$. En la década de los 70 la tendencia positiva se estancó, registrando un ligero aumento hasta llegar a un $41 \%$ en 1980. Luego del estallido de la crisis de la deuda y la puesta en marcha de las políticas de ajuste y estabilización, la regresión social cobró más fuerza: la proporción de pobres saltó al 43\% en 1986 y un $46 \%$ en 1990, esto es, 196 millones de latinoamericanos. En 1980, el número de pobres en la región era de unos 136 millones y ya en 1994 era 210 millones. Fuentes más recientes señalan que en 1999 había 224

24 Los eufemismos son muy empleados en el lenguaje políticamente correcto. Son comunes en la política para camuflar o hacer que suenen más naturales las políticas impopulares o una crisis económica. También se emplean eufemismos para suavizar blasfemias: en francés se tiene parbleu en lugar de par Dieu. En español tenemos diantre en vez de diablo y diez o sos sustituyendo a Dios en varias expresiones blasfemas. Lo contrario de un eufemismo es un disfemismo. millones de pobres en la región (no incluye al Caribe anglófono ${ }^{25}$ ).

- Un estudio de la Organización Panamericana de la Salud (OPS) y de la Organización Internacional del Trabajo (OIT), ha señalado que, a fines de 1999, 267 millones de latinoamericanos y caribeños sufrían exclusión de los servicios de salud relacionados con el déficit de camas en los hospitales; 152 millones no disponían de agua potable y/o alcantarillado y más de 125 millones estaban en condiciones de inaccesibilidad geográfica a los servicios de salud.

- La implantación de las recetas neoliberales en América Latina han dejado como saldo sociedades mucho más heterogéneas y fragmentadas, surcadas por profundas desigualdades de todo tipo: clase, etnia, género, religión, etc.

- Los indigentes eran 62 millones en 1980 y para 1994 ya sumaban 98 millones, por lo que el neoliberalismo se anotó 36 millones de indigentes. Ya en 1999 la cifra de indigentes se había elevado a 100 millones (no incluye al Caribe anglófono).

- $\quad$ El desempleo en 1990 era de 5,9\% y para 1998 había ascendido al 8,4 \%. A su vez, en el decenio de 1990 la mayor parte del empleo generado en América Latina y el Caribe correspondió al llamado sector informal.

- La concentración de la mano de obra en la producción ha disminuido, llevando a niveles pavorosos la brecha económica entre ricos y pobres.

- Se ha fortalecido el apego de los individuos a los bienes materiales y al olvido de problemas clave como la justicia social, la desigualdad o la calidad de vida.

25 Se aplica al territorio que está habitado por población de habla inglesa. 
- Se ha aumentado la corrupción, la drogadicción y la violencia a grados alarmantes.

- Para 1997 se calculó que 6 millones de niños y adolescentes de América Latina y el Caribe sufrían agresiones de diversos tipos, incluido abusos sexuales, y que alrededor de 80 mil morían anualmente como consecuencia de esas prácticas. También se estimó que entre 40 millones y 50 millones de menores de la región (solo entre 6 y 14 años) estaban condenados a intentar sobrevivir en las calles.

- Se ha intensificado la degradación ecológica ocasionada en buena medida por la reducción de los recursos destinados a la protección del entorno dentro del presupuesto de los gobiernos de la región.

- Los catastróficos resultados del neoliberalismo en el hemisferio exacerbaron con el transcurso del tiempo las acciones de los movimientos sociales que fueron cobrando cada vez más auge y prosélitos, al unísono que se buscaban alternativas al neoliberalismo. De esta manera, se fue articulando una nueva izquierda latinoamericana. Entre los ejemplos más ilustrativos del auge y la resistencia de los movimientos sociales frente al neoliberalismo podemos mencionar las pioneras revueltas de los zapatistas en 1994, contrarias a la firma del Tratado de Libre Comercio entre Estados Unidos, Canadá y México; la aparición de los piqueteros argentinos; el Movimiento Sin Tierra (MST) en Brasil; los indígenas cocaleros en Bolivia; el movimiento indígena en Ecuador; los estallidos sociales producidos en Ecuador en el 2000 y en Argentina en el 2001, que dieron al traste con los gobiernos neoliberales de Jamil Mahuad y Fernando de la Rúa, respectivamente; el entierro del Área de Libre Comercio de las Américas (ALCA) en Mar del Plata, Argentina, en noviembre del 2005, instrumento neoliberal que trató de imponer el gobierno de los Estados Unidos a los países de la región durante la celebración de la Cumbre de las Américas; las victorias electorales de Hugo Chávez en Venezuela en 1998, de Nestor Kirchner en Argentina en 2003, de Evo Morales en Bolivia en 2005, de Daniel Ortega en Nicaragua y Rafael Correa en Ecuador en 2006, entre otros que pudiéramos mencionar, demuestran que América Latina se ha encauzado por otros rumbos, abandonando paulatinamente el modelo neoliberal que tanto daño le ha causado a los pueblos latinoamericanos. Este proceso ha marchado en unos países con mayor celeridad que en otros, de acuerdo al contexto de cada cual y de la voluntad política de sus administraciones, fundamentalmente de la figura presidencial. En este proceso han sobresalido los presidentes Hugo Chávez y Evo Morales, quienes han surcado el camino, avalados por impresionantes y revolucionarios avances sociales hacia lo que se ha llamado el socialismo del siglo XXI.

- El neoliberalismo ha reforzado la idea de la integración latinoamericana como única alternativa posible para superar la difícil situación económica, política y social, acumulada por más de tres décadas de prácticas neoliberales, así como la única vía de enfrentar la hegemonía estadounidense en la región. La materialización de este proceso se ha hecho más notoria en el fortalecimiento del MERCOSUR y el surgimiento de la Alternativa Bolivariana para las Américas (ALBA), integrada inicialmente por Cuba y Venezuela, y a la que se sumaron posteriormente Bolivia y Nicaragua.

\section{Análisis y discusión}

La Teoría General del Equilibrio muestra muchas inconsistencias en sus teoremas y fundamentos. En la década de 1930 el equilibrio general ya era objeto de crítica, dando lugar al 
impulso de un modelo donde el Estado debía intervenir en el mercado.

El problema es que en la teoría económica, como se puede observar, prácticamente hay un subastador (mano invisible) que centraliza todo el proceso en el mercado, lo cual contradice toda la teoría del equilibrio general cuando se argumenta que el mercado no debe ser centralizado. El neoliberalismo ha querido vender la idea que el Estado no debe de intervenir en el mercado, aunque los resultados del neoliberalismo son adversos sobre las sociedades más desiguales.

Por insistencia de los economistas neoclásicos siempre se ha continuado con el desarrollo de la teoría del equilibrio, aunque a la conclusión que han llegado no ha sido del todo alentadora. De hecho, Kenneth Arrow insiste que "en general se alcanza el equilibrio", pero, ¿no será que esta es tan solo una conjetura? Probablemente debemos de reflexionar en que la teoría del equilibrio es la teoría de la imposibilidad.

Es probable que cualquier modelo económico capitalista cause efectos negativos sobre las sociedades pobres, y que uno de los más despiadados ha sido el neoliberal. Sin embargo, el modelo económico del Estado Benefactor no era tan perverso.

Por último, el capitalismo neoliberal está tomando a la humanidad como a una masa social que vende su fuerza de trabajo para generar riqueza a los más ricos, lo cual está dando como resultado la agudización de la pobreza y el detrimento del ambiente.

\section{Conclusiones}

- La Teoría General del Equilibrio es inconsistente porque necesita de supuestos que están sujetos a la subjetividad humana para alcanzar la eficiencia.

- $\quad$ El modelo económico dominante presenta inconsistencias y contradicciones en sus fundamentos teóricos; consecuentemente, el modelo de mercados abiertos produce efectos negativos sobre las sociedades más desiguales.

- La economía ambiental no ha sido la solución a los problemas e impactos que ha tenido el mercado sobre el ambiente, porque siempre se valora al ambiente en un sentido estrictamente económico, sin detener la degradación ambiental.

- En un mercado de un producto en el que existen impactos hacia el ambiente o "externalidades", bien sea en el consumo o en la producción, el mercado no asigna las cantidades eficientes del mismo.

- El mercado no da solución por sí solo a los problemas ambientales, por muy bien delimitados que estén los derechos de propiedad.

- El neoliberalismo ha fracasado desde el punto de vista económico, pues no ha conducido a ninguno de los países de la región al desarrollo.

- Se debe generar un nuevo modelo económico, retomando nuevamente la intervención del Estado para propiciar el pleno empleo, porque durante los últimos 30 años se ha demostrado que el libre mercado no armoniza las sociedades.

\section{Referencias}

Bonilla, G. 2007. "El fin del mundo", segunda parte. Revista cultural e informativa de Jalapa. Xalapán.

Coase, R.H., 1994. La estructura institucional de la producción. La empresa, el mercado y la ley. Alianza editorial.

Coase, R.H., 1960. "The Problem of Social Cost", en "Journal of Law and Economics", (3), 1). 
Eagly, R. 1968. Events, ideology and economic theory. Detroit, Wayne State University Press.

Funtowicz, S. 1994. Epistemología política, Ciencia con la gente. Buenos Aires, Argentina. Centro Editor de América Latina.

Grossman, G.M. and Krueger, A.B. (1993). Environmental Impacts of a North American Free Trade Agreement. In The Mexico-U.S. Free Trade Agreement, P. Garber, ed. Cambridge, Mass.: MIT Press.

Hessen, J. 1990. Teoría del Conocimiento. Segunda Edición. México. Editores Mexicanos Unidos.

Hobbes, T. 1987. Del ciudadano y Leviatán. Estudio preliminar y antología de Enrique Tierno Galván. Traducción de Enrique Tierno Galván y M. Sánchez Sarto. Madrid, España: Editorial Tecnos.

Larraín, M. 2004. Análisis crítico del Consenso de Washington (en línea). Consultado el 19 agosto 2008. Disponible en http:// www.propolco.cl/blog/wp- content/archivo/11sem/wa shington.htm - 16k -

Leff, E. 2004. Racionalidad Ambiental. México. Siglo XXI editores, S.A. 509 p.

Leff, E. 2007. Saber Ambiental. México. Siglo XXI editores, S.A. 414 p.

McAfee, K., 2003. Neoliberalism on the molecular scale. Economic and genetic reductionism in biotechnology battles (en línea). Consultado 23 jul 2008. Disponible en http://www.sciencedirect.com

Martínez, A.; Roca, J. 2003. Economía Ecológica y Política Ambiental. México, Fondo de Cultura Económica.

Martínez Allier, J. 2008. Primer Diplomado Latinoamericano sobre Economía Ecológi- ca. Facultad Latinoamericana de Ciencias Sociales. FLACSO-GUATEMALA.

Marx, K. 1867. El Capital, Libro III. Siglo XXI, Editores, S.A. Buenos Aires, Argentina.

Meadows, D. 1972. Los límites del crecimiento. Cambridge, Massachusetts. U.S.A.

Monterroso, N. 2007. Ciencia Social y Forestería Comunitaria. Diplomado Mesoamericano sobre Forestería Comunitaria. Facultad Latinoamericana de Ciencias Sociales. FLACSO- GUATEMALA.

Nadal, A. 2008. Teoría Económica Dominante. Primer Diplomado en Economía Ecológica. Facultad Latinoamericana en Ciencias Sociales. FLACSO-GUATEMALA.

Napoleoni, C. 1972. Economic thought of the twentieth century. Londres, England.

Ramírez, E. 2008. Neoliberalismo en América Latina: Orígenes, evolución y consecuencias (en línea). La Habana, Cuba. Consultado 19 agosto 2008. Disponible en http://www.lajiribilla. cu/2008/n379_08/379_08.html

Screpanti Ernesto, Zamagni Stefano 1997: Panorama de historia del pensamiento económico. Editorial Ariel, S.A. Barcelona.

Smith, A. 1776. La Causa y Naturaleza de la Riqueza de las Naciones. University of Glasgow.

Sorrell, T. 1986. Hobbes (Routledge \& Kegan Paul, London) - A concise and well- judged account of Hobbes's life and works.

Weber, M. 2006. La ética protestante y el espíritu del capitalismo. Madrid, España. Alianza Editorial, S.A.

Zaratiegui, J. 2002. "Alfred Marshall y la teoría económica del empresario", Valladolid, Spain, ISBN: 978-84-8448-139-3, 2002, Universidad de Valladolid. 